\title{
Sorafenib-induced Pancreatic Pseudocyst in a Patient with Advanced Hepatocellular Carcinoma: a Rare Adverse Event
}

\author{
Dae-ha Kim, Minkoo Kim, Hyung Joon Yim, Sang Jun Suh, Young Kul Jung \\ Division of Gastroenterology and Hepatology, Department of Internal Medicine, Korea University Ansan Hospital, Ansan, Korea
}

Received Feb. 23, 2018

Revised Sep. 21, 2018

Accepted Sep. 24, 2018
A 54-year old man diagnosed with advanced hepatocellular carcinoma began treatment with sorafenib. After 3 weeks of treatment, he complained of abdominal pain and nausea. Abdominal sonography showed multiple hepatic lesions only. Serum amylase and lipase levels were $35 \mathrm{U} / \mathrm{L}$ and $191 \mathrm{U} / \mathrm{L}$, respectively. The patient was diagnosed with sorafenib-induced acute pancreatitis. After 10 days of discontinuing sorafenib he still complained of nausea and loss of appetite. Esophagogastroduodenoscopy showed a large bulging lesion, which was suspected to cause extrinsic compression on the high body of the gastric anterior wall. Computed tomography scan revealed a cystic lesion, $8.3 \mathrm{~cm}$ in size, in the pancreatic tail, suggesting a pancreatic pseudocyst. After the withdrawal of sorafenib, systemic chemotherapy with Adriamycin and cisplatin was administered. Four months after the discontinuation of sorafenib, the size of the pancreatic pseudocyst decreased from $8.3 \mathrm{~cm}$ to $3 \mathrm{~cm}$. The patient's symptoms were also relieved. (J Liver Cancer 2019;19:154-158)

Keywords: Advanced hepatocellular carcinoma; Sorafenib; Adverse event; Pancreatic pseudocyst; Pancreatitis

\section{INTRODUCTION}

Sorafenib, a multi-tyrosine kinase inhibitor, is an orally administered, molecular targeted therapeutic agent that inhibits Raf kinase, vascular endothelial growth factor receptor, and platelet-derived growth factor receptor. It has been the only standard therapy that was proven to improve survival in advanced hepatocellular carcinoma before the introduction of newer molecular targeted therapeutic agents. ${ }^{1}$ The common side effects of sorafenib include systemic symptoms such as fatigue, skin manifestations such as hand-foot skin

\section{Corresponding author: Hyung Joon Yim}

Division of Gastroenterology and Hepatology, Department of Internal Medicine, Korea University Ansan Hospital, 123 Jeokgeum-ro, Danwongu, Ansan 15355, Korea

Tel. +82-31-412-6565, Fax. +82-31-412-5582

E-mail; gudwns21@korea.ac.kr

https://orcid.org/0000-0002-6036-2754 reaction, and alopecia. ${ }^{2}$ However, nausea, vomiting, and other common gastrointestinal symptoms are uncommon, and cases of acute pancreatitis are rare. ${ }^{3}$ In this article, we report a case of acute pancreatitis and pancreatic pseudocyst after sorafenib treatment in a patient with advanced hepatocellular carcinoma.

\section{CASE REPORT}

\section{Clinical and laboratory findings}

A 54-year-old man visited the emergency room for aggravated epigastric pain. The patient had chronic hepatitis B since his twenties and received no special treatment. He was previously diagnosed with hepatocellular carcinoma (2 lesions in segments 6 and 8 ) at the age of 46 and was transferred to our hospital. He received transarterial chemoembolization 
(TACE) and radiofrequency ablation. The hepatocellular carcinoma recurred in segment 5 after 3 years, and the patient received additional TACE followed by laparoscopic wedge resection. During subsequent follow-up, the patient was found to have recurrent multiple hepatocellular carcinoma with the continued elevation of alpha-fetoprotein, and the lesion continued to progress even after repeated TACEs in the last 1 year. He started $400 \mathrm{mg}$ of sorafenib twice a day 3 weeks ago. At the first outpatient visit after one week of sorafenib treatment, the patient complained of minor pain in the upper abdomen but the general condition was fair. He was instructed to revisit if the pain aggravated. As the pain worsened, the patient returned to the emergency room after discontinuing the medication for 2 days. The abdominal pain was assessed as 3 points on the Visual Analog Scale. The patient complained of nausea and indigestion in addition to abdominal pain, but there was no fever or diarrhea. As the abdominal pain worsened, the Eastern Cooperative Oncology Group Performance Status Scale worsened from 1 point to 3 points. Apart from hepatitis B and hepatocellular carcinoma, the patient had type 2 diabetes for which he was taking glimepiride. The patient was a social drinker, but abstained from alcohol since diagnosis of the hepatocellular carcinoma. Physical examination showed slight tenderness in the right upper abdomen and the epigastrium, but no rebound tenderness or abdominal rigidity was observed. The laboratory findings were as follows: leukocyte $5,480 / \mathrm{uL}$, hemoglobin $15.6 \mathrm{~g} / \mathrm{dL}$, platelet $301,000 / \mathrm{uL}$, total protein $7.0 \mathrm{~g} / \mathrm{dL}$, albumin $3.7 \mathrm{~g} / \mathrm{dL}$, aspartate aminotransferase $25 \mathrm{IU} / \mathrm{L}$, alanine aminotransferase $34 \mathrm{IU} / \mathrm{L}$, total bilirubin $1.65 \mathrm{mg} / \mathrm{dL}$, direct bilirubin $1.00 \mathrm{mg} /$ dL, gamma glutamyl transpeptidase 596 IU/L, alkaline phosphatase $350 \mathrm{IU} / \mathrm{L}$, amylase $35 \mathrm{U} / \mathrm{L}$, lipase $191 \mathrm{U} / \mathrm{L}, \mathrm{C}$-reactive protein $10.660 \mathrm{mg} / \mathrm{dL}$, blood urea nitrogen $16.8 \mathrm{mg} / \mathrm{dL}$, and creatinine $0.72 \mathrm{mg} / \mathrm{dL}$. In the coagulation test, the prothrombin time was 16.7 seconds (international normalized ratio 1.45). The creatine kinase-muscle/brain fraction and troponin T levels were normal, and the electrocardiography showed normal sinus rhythm. Abdominal ultrasonography showed a sludge-filled gallbladder and multiple masses with increased echogenicity in the liver, but there were no dilated bile ducts or pancreatic abnormalities.

\section{Management, progress, and final diagnosis}

After the initial examination, the patient's abdominal pain was suspected to be 1) acute gastritis due to sorafenib administration, 2) acute pancreatitis associated with sorafenib, or 3 ) acute biliary pancreatitis or cholangitis due to the gallbladder sludge which was either related or unrelated to sorafenib administration. We therefore decided to 1) discontinue sorafenib as a possible cause, 2) fast the patient, keeping in mind the possible aforementioned conditions and administer sufficient intravenous fluids, and 3) observe the improvement in clinical features and results of biochemical tests. We also intended to perform computed tomography (CT) scans in case the patient had fever or the symptoms or test results deteriorated. The patient improved to 1 point on the Visual Analog Scale system from the day after admission, and the levels of lipase, gamma glutamyl transpeptidase, and alkaline phosphatase decreased to $126 \mathrm{U} / \mathrm{L}, 426 \mathrm{IU} / \mathrm{L}$, and $257 \mathrm{IU} / \mathrm{L}$, respectively. On the 3rd day of admission, the patient no longer complained of abdominal pain and started having meals, while the levels of total bilirubin and C-reactive protein improved to $1.45 \mathrm{mg} / \mathrm{dL}$ and $8.620 \mathrm{mg} / \mathrm{dL}$, respectively. The patient remained stable, the abdominal pain resolved, and continued to have meals, but he continued to complain of intermittent nausea and indigestion despite the administration of proton pump inhibitors and pro-

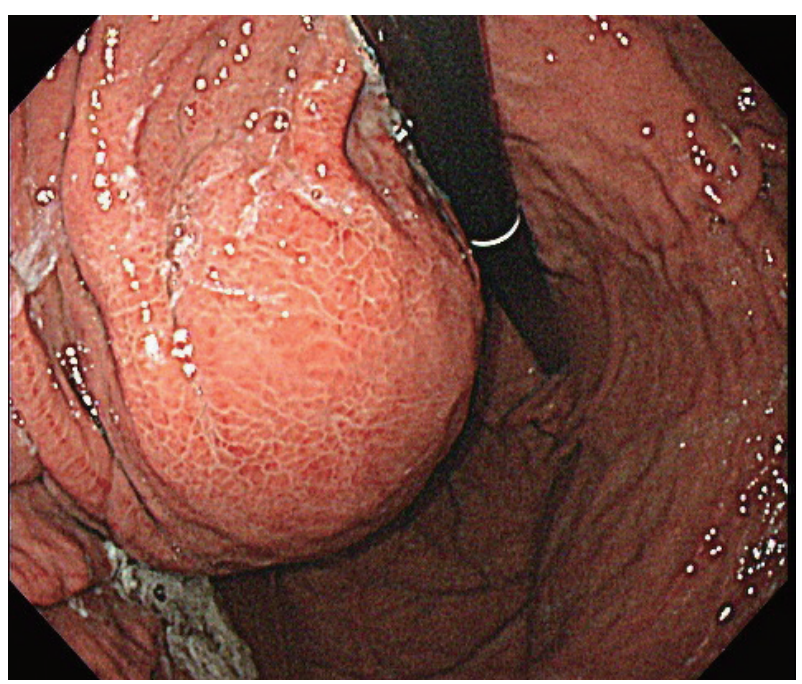

Figure 1. Endoscopic finding. Extrinsic compression was suspected from the endoscopic finding. 
kinetics. Endoscopy was additionally performed, and the endoscopic findings showed no significant changes in the esophageal varices and reflux esophagitis as well as portal hypertensive gastropathy compared to the findings 9 months ago. However, a new large bulging mass, occupying the gastric lumen from the anterior wall of the upper body to the fundus, was observed, and was suspected to cause extrinsic compression (Fig. 1). A CT scan of the abdomen revealed a new cystic lesion, about $8 \mathrm{~cm}$ in size, with necrosis of the pancreatic tail parenchyma, which was diagnosed as a pseudocyst of the pancreas (Fig. 2A, B). As the abdominal pain resolved and there were no signs of infection, the lesion was monitored for spontaneous resolution without further interventions.

\section{Follow-up outcomes}

The patient's condition improved to 1 point on the Eastern Cooperative Oncology Group Performance Status Scale system, the abdominal pain resolved, and his dietary intake recovered. Sorafenib was discontinued and the treatment was changed to Adriamycin and cisplatin systemic chemotherapy. One month later, the CT scan showed that the size of the pseudocyst in the pancreatic tail decreased to about $7 \mathrm{~cm}$. It was planned that the patient would maintain systemic che-
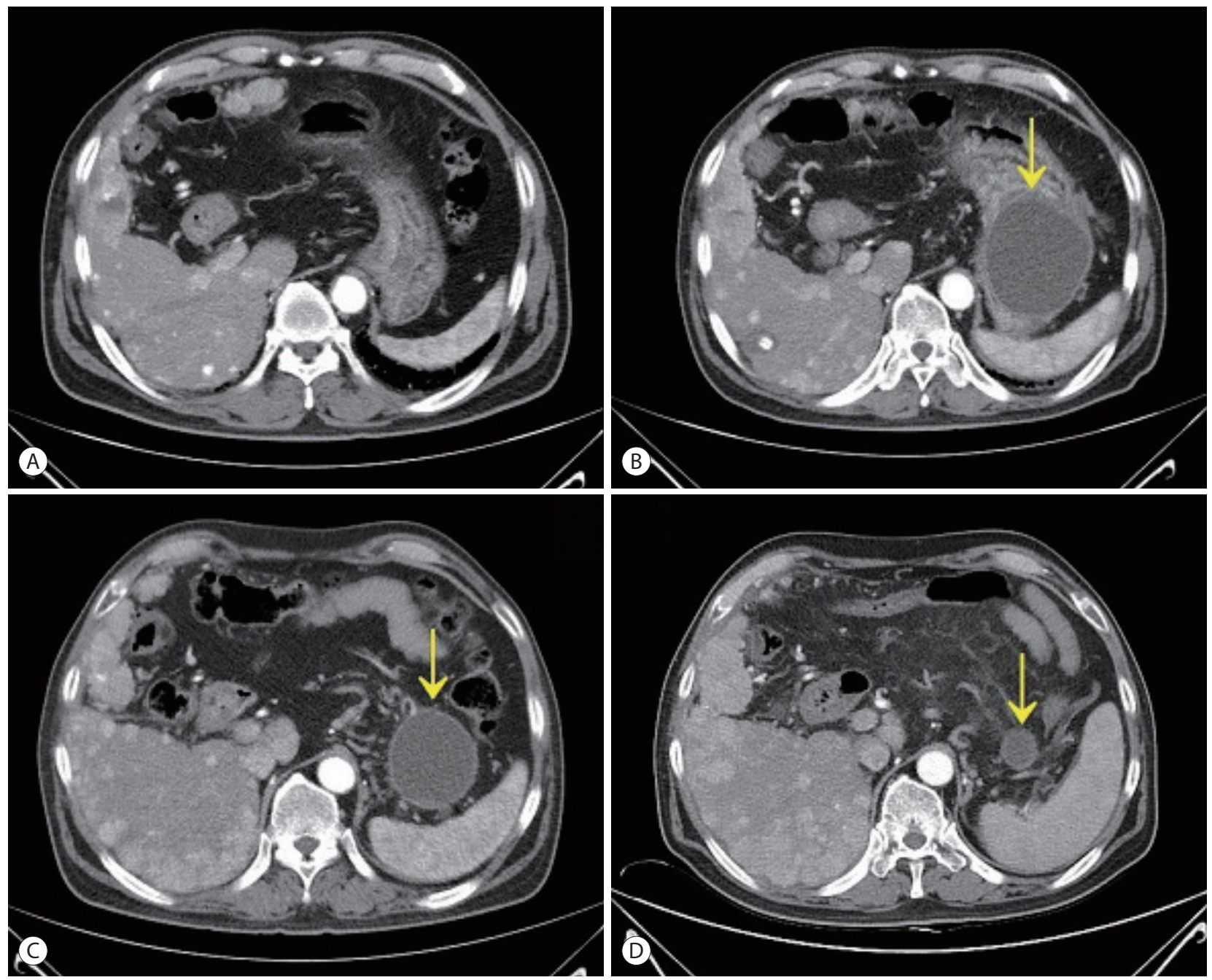

Figure 2. Serial computed tomography (CT) scans. CT scan obtained 2 months before sorafenib administration (A). CT scans showed a newly developed pancreatic pseudo cyst (arrow) after 3 weeks of treatment with sorafenib (B) and regression of the lesion 2 months (C) and 4 months (D) after the discontinuation of sorafenib. 
motherapy and follow-up the pancreatic lesion at regular treatment response evaluations (Fig. 2C, D).

\section{DISCUSSION}

The levels of lipase and amylase are elevated in about 30$40 \%$ of patients after taking sorafenib, but acute pancreatitis is a rare occurrence, with $<1 \%$ incidence. ${ }^{4}$ It is to be noted that only a few sporadic cases have been reported to date. The mechanism by which sorafenib induces pancreatitis has been linked to the anti-vascular endothelial growth factor activity of sorafenib. This leads to ischemia of the acinar cells, resulting in acinar cell apoptosis. ${ }^{7,8}$ It has also been argued that sorafenib causes pancreatitis by causing backflow of the contents of the duodenum into the pancreatic duct. ${ }^{5}$

Although pancreatic pseudocyst is a possible complication of acute pancreatitis, there have been no reported cases of pancreatic pseudocyst formation as a complication of acute pancreatitis caused by sorafenib. Except sorafenib, other types of tyrosine kinase inhibitors, including crizotinib and pazopanib, have been reported to induce the formation of pseudocysts along with acute pancreatitis. ${ }^{9,10}$ However, this is the first case of pancreatic pseudocyst formation following sorafenib treatment in advanced hepatocellular carcinoma.

Generally, drug-induced pancreatitis is diagnosed by a clear change in the clinical features depending on the time of administration and discontinuation of the drug. Additionally, the other causes of pancreatitis should be excluded. In this case, the symptoms began when the patient started taking sorafenib, and the symptoms slightly improved after discontinuation of the drug, but nausea and indigestion persisted. The patient was subsequently diagnosed with a pseudocyst resulting as a complication of pancreatitis, 4 weeks after the first symptom appeared. This is consistent with the fact that the associated complications usually appear more than four weeks after the onset of pancreatitis. ${ }^{11}$

In this case, it was difficult to rule out the possibilities of both cholangitis and pancreatitis being associated with the sludge, because the sludge was observed in the gallbladder on ultrasonography. However, we did not observe any abnormalities in the bile ducts and pancreas on CT, and there was no obstructive jaundice or fever, which are common in cholangitis. If the results of a biochemical test continued to worsen, such as the levels of bilirubin or pancreatic enzymes elevated, even after the discontinuation of sorafenib, then either magnetic resonance cholangiopancreatography or endoscopic retrograde cholangiopancreatography would have to be performed for evaluating any diseases in the bile duct.

In this case, the pseudocyst was not detected by abdominal ultrasonography at the time of the first visit to the emergency room. This may be due to the differences in sensitivity between ultrasound and CT in pancreatic diseases rather than the possibility of lesions occurring within a week. ${ }^{12,13}$

Most cases of acute pancreatitis reported so far have shown improvement upon discontinuation of the causative agent and administration of conservative treatment. However, pseudocysts may lead to fatal consequences such as secondary infections, complications such as peritonitis due to ruptured cysts, and formation of pseudoaneurysms and bleeding, requiring clinicians to pay attention during treatment with sorafenib.

In conclusion, this is a rare case of acute pancreatitis and pancreatic pseudocyst caused by sorafenib treatment in a patient with advanced hepatocellular carcinoma. It should be kept in mind that these complications are very rare but can be fatal in some cases. In patients with elevated pancreatic enzymes and clinical symptoms suggestive of acute pancreatitis, CT imaging rather than ultrasonography should provide a more accurate and faster diagnosis.

\section{ACKNOWLEDGEMENT}

This study was funded by Korea University Grants.

\section{Conflicts of Interest}

The authors have no conflicts to disclose.

\section{REFERENCES}

1. Liu L, Cao Y, Chen C, Zhang X, McNabola A, Wilkie D, et al. Sorafenib blocks the RAF/MEK/ERK pathway, inhibits tumor angiogenesis, and induces tumor cell apoptosis in hepatocellular 
carcinoma model PLC/PRF/5. Cancer Res 2006;66:11851-11858.

2. Llovet JM, Ricci S, Mazzaferro V, Hilgard P, Gane E, Blanc JF, et al. Sorafenib in advanced hepatocellular carcinoma. N Engl J Med 2008;359:378-390.

3. Li M, Srinivas S. Acute pancreatitis associated with sorafenib. South Med J 2007;100:909-911.

4. Pezzilli R, Corinaldesi R, Morselli-Labate AM. Tyrosine kinase inhibitors and acute pancreatitis. JOP 2010;11:291-293.

5. Amar S, Wu KJ, Tan WW. Sorafenib-induced pancreatitis. Mayo Clin Proc 2007;82:521.

6. Kobayashi Y, Kanemitu T, Kamoto A, Satoh M, Mori N, Sekii K, et al. Painless acute pancreatitis associated with sorafenib treatment: a case report. Med Oncol 2011;28:463-465.

7. Chou JW, Cheng KS, Huang CW. Sorafenib-induced acute pancreatitis: a case report and review of the literature. Intern Med 2016;55:623-627.

8. Ratain MJ, Eisen T, Stadler WM, Flaherty KT, Kaye SB, Rosner GL, et al. Phase II placebo-controlled randomized discontinuation trial of sorafenib in patients with metastatic renal cell carcinoma. J Clin
Oncol 2006;24:2505-2512.

9. Ishida H, Ichikawa W, Sasaki Y. Crizotinib-induced pancreatic pseudocyst: a novel adverse event. BMJ Case Rep 2015;2015.

10. Kawakubo K, Hata H, Kawakami H, Kuwatani M, Kawahata S, Kubo K, et al. Pazopanib-induced severe acute pancreatitis. Case Rep Oncol 2015;8:356-358.

11. Banks PA, Bollen $T L$, Dervenis $C$, Gooszen HG, Johnson CD, Sarr $M G$, et al. Classification of acute pancreatitis--2012: revision of the Atlanta classification and definitions by international consensus. Gut 2013;62:102-111.

12. Foley WD, Stewart ET, Lawson TL, Geenan J, Loguidice J, Maher $L$, et al. Computed tomography, ultrasonography, and endoscopic retrograde cholangiopancreatography in the diagnosis of pancreatic disease: a comparative study. Gastrointest Radiol 1980;5:2935.

13. Kressel HY, Margulis AR, Gooding GW, Filly RA, Moss A, Korobkin M. CT scanning and ultrasound in the evaluation of pancreatic pseudocysts: a preliminary comparison. Radiology 1978;126:153157. 